\title{
Asymptotic behaviour of pion-pion total cross-sections
}

\author{
David Greynat, ${ }^{a}$ Eduardo de Rafael ${ }^{b, c}$ and Grégory Vulvert ${ }^{d}$ \\ ${ }^{a}$ Dipartimento di Scienze Fisiche, Universita di Napoli "Federico II", \\ Via Cintia, 80126 Napoli, Italia \\ ${ }^{b}$ Aix-Marseille Université, CNRS, \\ CPT, UMR 7332, 13288 Marseille, France \\ ${ }^{c}$ Université de Toulon, CNRS, \\ CPT, UMR 7332, 83957 La Garde, France \\ ${ }^{d}$ Departament de Física Teórica, IFIC, \\ CSIC - Universitat de València, Apt. Correus 22085, E-46071 València, Spain \\ E-mail: david.greynat@gmail.com, EdeR@cpt.univ-mrs.fr, \\ vulvert@ific.uv.es
}

Abstract: We derive a sum rule which shows that the Froissart-Martin bound for the asymptotic behaviour of the $\pi \pi$ total cross sections at high energies, if modulated by the Lukaszuk-Martin coefficient of the leading $\log ^{2} s$ behaviour, cannot be an optimal bound in QCD. We next compute the total cross sections for $\pi^{+} \pi^{-}, \pi^{ \pm} \pi^{0}$ and $\pi^{0} \pi^{0}$ scattering within the framework of the constituent chiral quark model $(\mathrm{C} \chi \mathrm{QM})$ in the limit of a large number of colours $N_{c}$ and discuss their asymptotic behaviours. The same $\pi \pi$ cross sections are also discussed within the general framework of Large- $N_{c}$ QCD and we show that it is possible to make an Ansatz for the isospin $I=1$ and $I=0$ spectrum which satisfy the FroissartMartin bound with coefficients which, contrary to the Lukaszuk-Martin coefficient, are not singular in the chiral limit and have the correct Large- $N_{c}$ counting. We finally propose a simple phenomenological model which matches the low energy behaviours of the $\sigma_{\pi^{ \pm}}^{\text {total }} \pi^{0}(s)$ cross section predicted by the $\mathrm{C} \chi \mathrm{QM}$ with the high energy behaviour predicted by the Large- $N_{c}$ Ansatz. The magnitude of these cross sections at very high energies is of the order of those observed for the $p p$ and $p \bar{p}$ scattering total cross sections.

KeYwords: QCD Phenomenology, Phenomenological Models

ARXiv EPRINT: 1312.2881 


\section{Contents}

1 Introduction 1

2 Pion-pion amplitudes and sum rules 2

2.1 The Lukaszuk-Martin bound 4

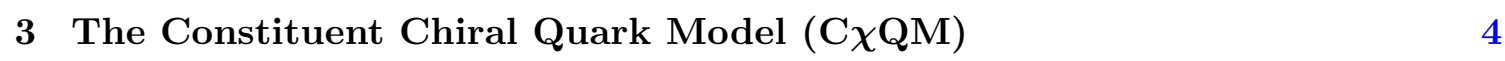

$\begin{array}{ll}3.1 \text { Calculation of the pion-pion total cross sections } & 7\end{array}$

$\begin{array}{lll}3.1 .1 & \text { The } \pi^{+} \pi^{-} \text {total cross section } & 7\end{array}$

$\begin{array}{lll}3.1 .2 & \text { The } \pi^{ \pm} \pi^{0} \text { total cross sections } & 8\end{array}$

3.1.3 The $\pi^{0} \pi^{0}$ total cross section 9

$\begin{array}{lll}3.2 & \text { Sum rules in the } \mathrm{C} \chi \mathrm{QM} & 10\end{array}$

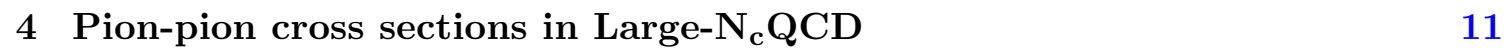

5 Numerical results and conclusions $\quad 14$

\section{Introduction}

The Froissart-Martin bound for the asymptotic behaviour of total cross sections has played a major role in the history of strong interactions. Using the Mandelstam representation, Froissart showed [1] that the total cross section $\sigma_{A B}^{\text {total }}(s)$ for the scattering of two hadronic particles $A$ and $B$ with center of mass energy $\sqrt{s}$ cannot grow faster than

$$
\sigma_{A B}^{\text {total }}(s) \underset{s \rightarrow \infty}{\leq} C \log ^{2} \frac{s}{s_{0}},
$$

with $C$ and $s_{0}$ unknown constants. The rigorous proof of this bound from axiomatic quantum field theory was later shown by Martin [2] and, quite remarkably, an explicit form for the coefficient $C$ was first derived by Lukaszuk and Martin in ref. [3] with the result

$$
C=\frac{4 \pi}{t_{0}}
$$

where $t_{0}$ denotes the lowest mass squared singularity in the $t$-channel of the scattering process. ${ }^{1}$ When applied to pion-pion scattering the Froissart-Martin-Lukaszuk bound (FMLbound) states that

$$
\sigma_{\pi \pi}^{\mathrm{tot}}(s) \underset{s \rightarrow \infty}{\leq} \frac{\pi}{m_{\pi}^{2}} \log ^{2} \frac{s}{s_{0}} .
$$

The presence of a factor $1 / m_{\pi}^{2}$ in the r.h.s. of eq. (1.3) has recently been questioned by two of us [5]: what happens to the FML-bound in $Q C D$ in the chiral limit when the

\footnotetext{
${ }^{1}$ With some assumptions, the normalization $s_{0}$ for averaged total cross sections has also been recently derived in ref. [4].
} 
pions, the Nambu-Goldstone states of the chiral-SU(2) flavour symmetry of QCD, become massless? Does the FML-bound become irrelevant in this limit as eq. (1.3) seems to indicate? As pointed out in ref. [5], the usual derivation of the FML-bound from the rigorous principles of axiomatic quantum field theory does not take into account the fact that the underlying dynamics of the strong interactions has the property of spontaneous chiral symmetry breaking. In fact, it implicitly assumes a realization of the hadronic spectrum $\grave{a}$ la Wigner-Weyl without Nambu-Goldstone particles, in which case, the coefficient of the $\log ^{2} s$ in eq. (1.3) is perhaps not so surprising; but what is then the correct coefficient in QCD? In the next section we derive a sum rule which clearly shows that the FML-bound cannot be the optimal bound in QCD.

Another question which was also raised in ref. [5] is: what becomes of the FML-bound in the QCD Large- $N_{c}$ limit? The Large- $\mathrm{N}_{\mathrm{c}}$ counting rules fix $\sigma_{\pi \pi}^{\text {tot }}(s)$ to be of $\mathcal{O}\left(1 / \mathrm{N}_{\mathrm{c}}\right)$, while the r.h.s. of eq. (1.3) appears to be of $\mathcal{O}(1)$. We propose here, as a very modest step towards an answer to these fundamental questions, to examine them first in the simple

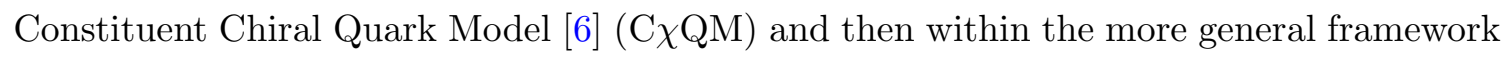
of Large- $\mathrm{N}_{\mathrm{c}}$ QCD properties.

We have organized this paper in the following way. In the next section we reproduce basic properties of the elastic pion-pion scattering amplitudes which are necessary to derive the sum rule which we have mentioned above. The effective Lagrangian of the $\mathrm{C} \chi \mathrm{QM}$ is described in section 3 as well as the results of the calculation of the total $\pi \pi$ annihilation cross sections in this model. We also show that the results obtained for these cross sections satisfy the sum rules which, within the model, fix some of the $\mathcal{O}\left(p^{4}\right)$ low-energy constants of the chiral Lagrangian. Section 4 is then dedicated to the pion-pion total cross sections in the QCD Large- $\mathrm{N}_{\mathrm{c}}$ limit where we reconsider in more detail some of the issues discussed in ref. [5] and where we propose a model which matches the low energy behaviours of the $\sigma_{\pi \pi}^{\text {total }}(s)$ cross sections predicted by the $\mathrm{C} \chi \mathrm{QM}$ with the high energy behaviours predicted by a simple Large- $\mathrm{N}_{\mathrm{c}}$ Ansatz. Phenomenological comments and conclusions are summarized in section 5 .

\section{Pion-pion amplitudes and sum rules}

In full generality, elastic $\pi \pi$ scattering in the isospin symmetry limit is described by a single invariant Lorentz amplitude $A(s, t, u) .^{2}$

$$
\begin{aligned}
&\left.\left\langle\pi^{d}\left(p_{4}\right) \pi^{c}\left(p_{3}\right) \text { out }\right| \pi^{a}\left(p_{1}\right) \pi^{b}\left(p_{2}\right) \text { in }\right\rangle \\
&=\mathbf{1}+i(2 \pi)^{4} \delta^{4}\left(p_{3}+p_{4}-p_{1}-p_{2}\right) \times \\
& \times\left\{\delta^{a b} \delta^{c d} A(s, t, u)+\delta^{a c} \delta^{b d} A(t, u, s)+\delta^{a d} \delta^{b c} A(u, s, t)\right\}
\end{aligned}
$$

where $a, b, c, d$ denote the 1,2,3 components of the adjoint representation of the pion fields in $\mathrm{SU}(2)$ and $s, t$ and $u$ the usual Mandelstam variables constrained by

$$
s+t+u=4 m_{\pi}^{2} .
$$

\footnotetext{
${ }^{2}$ For a modern review see ref. [7].
} 
It is convenient to work with the three $s$-channel isospin components $\mathbf{T}=\left(T^{0}, T^{1}, T^{2}\right)$ of the amplitudes in eq. (2.1):

$$
\begin{aligned}
& T^{0}(s, t)=3 A(s, t, u)+A(t, u, s)+A(u, s, t) \\
& T^{1}(s, t)=A(t, u, s)-A(u, s, t) \\
& T^{2}(s, t)=A(t, u, s)+A(u, s, t)
\end{aligned}
$$

These isospin amplitudes obey fixed- $t$ dispersion relations. They are the so-called Roy equations [8] which we shall consider at $t=0$ and in the chiral limit where $m_{\pi} \rightarrow 0$. The linear combination of the isospin amplitudes which diagonalize the crossing matrix in the Roy equations are then:

$$
\begin{aligned}
& F_{1}(s, 0)=-\frac{1}{6} T^{0}(s, 0)-\frac{1}{4} T^{1}(s, 0)+\frac{5}{12} T^{2}(s, 0) \\
& F_{2}(s, 0)=+\frac{1}{6} T^{0}(s, 0)+\frac{1}{4} T^{1}(s, 0)+\frac{7}{12} T^{2}(s, 0) \\
& F_{3}(s, 0)=-\frac{1}{6} T^{0}(s, 0)+\frac{3}{4} T^{1}(s, 0)+\frac{5}{12} T^{2}(s, 0) .
\end{aligned}
$$

The amplitudes $F_{2}$ and $F_{3}$ obey the same dispersion relation:

$$
\operatorname{Re} F_{2,3}(s, 0)=s^{2} \int_{0}^{\infty} \frac{\mathrm{d} s^{\prime 2}}{s^{\prime 2}} \frac{1}{s^{\prime 2}-s^{2}} \frac{1}{\pi} \operatorname{Im} F_{2,3}\left(s^{\prime}, 0\right),
$$

and Re $F_{2,3}(s, 0)$ are even under $s \leftrightarrow-s$, while the amplitude $F_{1}(s, 0)$ obeys the dispersion relation:

$$
\operatorname{Re} F_{1}(s, 0)=-\frac{s}{f_{\pi}^{2}}+2 s^{3} \int_{0}^{\infty} \frac{\mathrm{d} s^{\prime}}{s^{\prime 2}} \frac{1}{s^{\prime 2}-s^{2}} \frac{1}{\pi} \operatorname{Im} F_{1}\left(s^{\prime}, 0\right),
$$

and $\operatorname{Re} F_{1}(s, 0)$ is odd under $s \leftrightarrow-s$. Indeed, one can check that there is no contribution of $\mathcal{O}\left(s^{2}\right)$ to $\operatorname{Re} F_{1}(s, 0)$ in Chiral Perturbation Theory $(\chi \mathrm{PT})$, while the contributions of that order from $\chi \mathrm{PT}$ to the $F_{2}(s, 0)$ and $F_{3}(s, 0)$ amplitudes are [9]:

$$
\begin{aligned}
& \operatorname{Re} F_{2}(s, 0) \underset{s \rightarrow 0}{=} \frac{s^{2}}{f_{\pi}^{4}}\left[2 l_{1}^{\mathrm{r}}(\mu)+3 l_{2}^{\mathrm{r}}(\mu)+\frac{1}{12 \pi^{2}}\left(\log \frac{\mu^{2}}{s}+\frac{25}{24}\right)\right]+\mathcal{O}\left(s^{4}\right), \\
& \operatorname{Re} F_{3}(s, 0) \underset{s \rightarrow 0}{=} \frac{s^{2}}{f_{\pi}^{4}}\left[-2 l_{1}^{\mathrm{r}}(\mu)+l_{2}^{\mathrm{r}}(\mu)+\frac{1}{96 \pi^{2}}\right]+\mathcal{O}\left(s^{4}\right) .
\end{aligned}
$$

These $\chi \mathrm{PT}$ one-loop results are renormalization $\mu$-scale independent and well defined in the chiral limit. The $\mu$-scale dependence of the chiral log cancels with the $\mu$-dependence in the renormalized constants and the combination $-2 l_{1}^{\mathrm{r}}(\mu)+l_{2}^{\mathrm{r}}(\mu)$ is $\mu$-scale independent. The relation between the chiral SU(2) $l_{i}^{\mathrm{r}}$ constants and the more conventional $L_{i}^{\mathrm{r}}$ constants of the chiral $\mathrm{SU}(3)$ Lagrangian is as follows [10]:

$$
\begin{aligned}
& l_{1}^{\mathrm{r}}(\mu)=4 L_{1}^{\mathrm{r}}(\mu)+2 L_{3}-\frac{1}{96 \pi^{2}} \frac{1}{8}\left(\log \frac{M_{K}^{2}}{\mu^{2}}+1\right), \\
& l_{2}^{\mathrm{r}}(\mu)=4 L_{2}^{\mathrm{r}}(\mu)-\frac{1}{96 \pi^{2}} \frac{1}{4}\left(\log \frac{M_{K}^{2}}{\mu^{2}}+1\right),
\end{aligned}
$$


where here, kaon particles have been treated as massive and integrated out, hence the dependence on their mass $M_{K}$.

The optical theorem relates the amplitudes $\operatorname{Im} F_{i}(s, 0)$ to the total $\pi \pi$ cross sections as follows (massless pions):

$$
\begin{aligned}
& \operatorname{Im} F_{1}(s, 0)=\frac{1}{2}\left[s \sigma_{\pi^{+} \pi^{+}}^{\mathrm{tot}}-s \sigma_{\pi^{+} \pi^{-}}^{\mathrm{tot}}\right], \\
& \operatorname{Im} F_{2}(s, 0)=\frac{1}{2}\left[s \sigma_{\pi^{+} \pi^{+}}^{\mathrm{tot}}+s \sigma_{\pi^{+} \pi^{-}}^{\mathrm{tot}}\right]=\frac{1}{2}\left[s \sigma_{\pi^{ \pm} \pi^{0}}^{\mathrm{tot}}+s \sigma_{\pi^{0} \pi^{0}}^{\mathrm{tot}}\right], \\
& \operatorname{Im} F_{3}(s, 0)=\frac{1}{2}\left[3 s \sigma_{\pi^{ \pm} \pi^{0}}^{\mathrm{tot}}-s \sigma_{\pi^{0} \pi^{0}}^{\mathrm{tot}}\right] .
\end{aligned}
$$

\subsection{The Lukaszuk-Martin bound}

We are now in the position to explain what is the problem with the Lukaszuk-Martin coefficient [3] of the FML-bound in eq. (1.3) as well as with the recent averaged bound derived in ref. [4]. For that, let us consider the sum of eqs. (2.5) at small $s$, say $s=m_{\pi}^{2}$ to be precise, which we rewrite in the form of a convenient sum rule:

$$
\frac{\pi}{f_{\pi}^{4}}\left[l_{2}^{\mathrm{r}}(\mu)+\frac{1}{48 \pi^{2}}\left(\log \frac{\mu^{2}}{m_{\pi}^{2}}+\frac{7}{6}\right)\right]+\mathcal{O}\left(m_{\pi}^{4}\right)=\int_{4 m_{\pi}^{2}}^{\infty} \frac{\mathrm{d} s^{\prime}}{s^{\prime 2}-m_{\pi}^{4}} \sigma_{\pi^{ \pm} \pi^{0}}^{\mathrm{tot}}\left(s^{\prime}\right),
$$

where on the 1.h.s. we have used eqs. (2.7), (2.8) and on the r.h.s. the fact that

$$
\operatorname{Im} F_{2}\left(s^{\prime}, 0\right)+\operatorname{Im} F_{3}\left(s^{\prime}, 0\right)=2 s^{\prime} \sigma_{\pi^{ \pm} \pi^{0}}^{\text {tot }}\left(s^{\prime}\right) .
$$

We recall that the l.h.s. does not depend on the choice of the renormalization scale $\mu$. Using the FML-bound we can write the integral in the r.h.s. as follows:

$$
\begin{aligned}
\int_{4 m_{\pi}^{2}}^{\infty} \frac{\mathrm{d} s^{\prime}}{s^{\prime 2}-m_{\pi}^{4}} \sigma_{\pi^{ \pm} \pi^{0}}^{\mathrm{tot}}\left(s^{\prime}\right) \leq \int_{4 m_{\pi}^{2}}^{s_{0}} & \frac{\mathrm{d} s^{\prime}}{s^{\prime 2}-m_{\pi}^{4}} \sigma_{\pi^{ \pm} \pi^{0}}^{\mathrm{tot}}\left(s^{\prime}\right) \\
& +\frac{\pi}{m_{\pi}^{2}} \frac{2}{s_{0}}\left[1+\frac{1}{27} \frac{m_{\pi}^{4}}{s_{0}^{2}}+\mathcal{O}\left(\frac{m_{\pi}^{4}}{s_{0}^{2}}\right)^{2}\right],
\end{aligned}
$$

where $s_{0}$ is the finite threshold where the asymptotic behaviour sets in, the same $s_{0}$ which appears as the normalization of the $\log ^{2} s$ in the FML bound in eq. (1.3). Both terms in the r.h.s. of this inequality are obviously positive. Inserting this inequality in the r.h.s. of the sum rule in eq. (2.12) shows that the 1.h.s., which goes as $\log m_{\pi}^{2}$ in the chiral limit, is bounded by a quantity which diverges as $1 / m_{\pi}^{2}$ in the same limit. We therefore conclude that, if the Froissart bound applies to $\pi \pi$ scattering in QCD, the Lukaszuk-Martin coefficient of the leading $\log ^{2} s$ term cannot be the optimal one.

We wish to emphasize that the derivation of this result follows from very general properties of $\pi \pi$ amplitudes in QCD and, so far, we have not consider the Large- $\mathrm{N}_{\mathrm{c}}$ approximation.

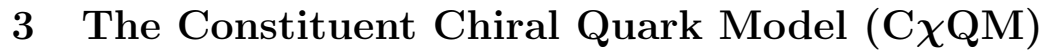

Historically, the model in question emerged as an attempt to reconcile the successes of phenomenological quark models, like e.g. the De Rújula-Georgi-Glashow model [11], with 
QCD. The Lagrangian proposed by Manohar and Georgi (MG) is an effective field theory which incorporates the interactions of the low-lying pseudoscalar particles of the hadronic spectrum i.e., the Nambu-Goldstone modes of the spontaneously broken chiral symmetry [12], with chirally rotated quark fields $Q=(U, D, S)$. These quarks have become massive due to the phenomenon of spontaneous chiral symmetry breaking ( $\chi \chi \mathrm{SB}$ ). Their mass, however, $\left(M_{Q} \sim \frac{1}{3} M_{\text {nucleon }}\right)$ has nothing to do with the masses of the $u, d, s$ quark fields in the QCD Lagrangian which explicitly break chiral symmetry and are known to be much smaller (see e.g. the recent review article in ref. [13]). The constituent quark fields may also have gluonic interactions but, since the Goldstone modes are already in the Lagrangian, the color-SU(3) coupling constant is then supposed to be no longer running and relatively small. The hope is that such an effective Lagrangian encodes the essential degrees of freedom to describe Hadron Physics at energies below the chiral symmetry breaking scale but still above the confinement regime.

It is fair to say, however, that in spite of some efforts (see e.g. refs. [14-16] and references therein), it has not been possible to establish the approximations at which the MG-Lagrangian could be derived from the underlying QCD theory. It can be shown to be a particular case of the Extended Nambu Jona-Lasinio (ENJL) Model [17, 18], but this only transfers the problem of its derivation from first principles to another level where, in any case, the basic question remains so far unanswered.

An interesting observation made by Weinberg [15] is the fact that in the limit of a large number of colors $\mathrm{N}_{\mathrm{c}}$ the $\mathrm{C} \chi \mathrm{QM}$ becomes a renormalizable theory. A subsequent observation along the same line was made in ref. [19] where it is shown that the number of counterterms which in the Large $-\mathrm{N}_{\mathrm{c}}$ limit have to be added to the primitive ManoharGeorgi Lagrangian, is minimized for the choice $g_{A}=1$. In that respect, it has also been shown $[19,20]$ that there is a class of observables governed by integrals of specific QCD Green's functions which, for $g_{A}=1$, have rather good matching to their short-distance behaviour. Interesting examples are the Hadronic Vacuum Polarization, the Hadronic Light-by-Light Scattering and the Hadronic Electroweak contributions to the anomalous magnetic moment of the muon, which have been recently discussed within the framework of the C $\chi$ QM in ref. [20].

The effective Lagrangian in question is the following:

$$
\begin{aligned}
\mathcal{L}_{\mathrm{C} \chi \mathrm{QM}}(x)= & \underbrace{i \bar{Q} \gamma^{\mu}\left(\partial_{\mu}+\Gamma_{\mu}+i G_{\mu}\right) Q+\frac{i}{2} g_{A} \bar{Q} \gamma^{\mu} \gamma_{5} \xi_{\mu} Q-M_{Q} \bar{Q} Q}_{M-G}-\frac{1}{2} \bar{Q}\left(\Sigma-\gamma_{5} \Delta\right) Q \\
& +\underbrace{\frac{1}{4} f_{\pi}^{2} \operatorname{tr}\left[D_{\mu} U D^{\mu} U^{\dagger}\right.}_{M-G}+U^{\dagger} \chi+\chi^{\dagger} U]-\underbrace{\frac{1}{4} \sum_{a=1}^{8} G_{\mu \nu}^{(a)} G^{(a) \mu \nu}}_{M-G}+e^{2} C \operatorname{tr}\left(Q_{R} U Q_{L} U^{\dagger}\right) \\
& +L_{5} \operatorname{tr} D_{\mu} U^{\dagger} D^{\mu} U\left(\chi^{\dagger} U+U^{\dagger} \chi\right)+L_{8} \operatorname{tr}\left(U \chi \dagger U \chi \dagger+U^{\dagger} \chi U^{\dagger} \chi\right) .
\end{aligned}
$$

The underbraced terms are those of the MG-Lagrangian, but in the presence of external $\mathrm{SU}(3)$ vector $v_{\mu}(x)$ and axial-vector $a_{\mu}(x)$ sources. The field matrix $U(x)$ denotes the $3 \times 3$ unitary matrix in the flavour space which collects the Nambu-Goldstone fields and which 
under chiral rotations transforms as $U \rightarrow V_{R} U V_{L}^{\dagger}$. The vector field matrix $D_{\mu} U$ is the covariant derivative of $U$ :

$$
D_{\mu} U=\partial_{\mu} U-i r_{\mu} U+i U l_{\mu}, \quad l_{\mu}=v_{\mu}-a_{\mu}, \quad r_{\mu}=v_{\mu}+a_{\mu},
$$

and, with $U=\xi \xi$,

$$
\Gamma_{\mu}=\frac{1}{2}\left[\xi^{\dagger}\left(\partial_{\mu}-i r_{\mu}\right) \xi+\xi\left(\partial_{\mu}-i l_{\mu}\right) \xi^{\dagger}\right], \quad \xi_{\mu}=i\left[\xi^{\dagger}\left(\partial_{\mu}-i r_{\mu}\right) \xi-\xi\left(\partial_{\mu}-i l_{\mu}\right) \xi^{\dagger}\right] .
$$

The gluon field matrix in the fundamental representation of color $\mathrm{SU}(3)$ is $G_{\mu}(x)$ and $G_{\mu \nu}^{(a)}(x)$ its corresponding gluon field strength tensor. The presence of external scalar $s(x)$ and pseudoscalar $p(x)$ sources induces the extra terms proportional to

$$
\chi=2 B[s(x)+i p(x)]
$$

where $B$, like $f_{\pi}$, are order parameters not fixed by the model. When the $s(x)$ and $p(x)$ sources are frozen to the up, down, and strange light quark masses of the QCD Lagrangian,

$$
\chi=2 B \mathcal{M}, \quad \text { with } \quad \mathcal{M}=\operatorname{diag}\left(m_{u}, m_{d}, m_{s}\right),
$$

and then

$$
\Sigma=\xi^{\dagger} \mathcal{M} \xi^{\dagger}+\xi \mathcal{M}^{\dagger} \xi, \quad \Delta=\xi^{\dagger} \mathcal{M} \xi^{\dagger}-\xi \mathcal{M}^{\dagger} \xi
$$

With the axial coupling fixed to $g_{A}=1$, the extra couplings $L_{5}$ and $L_{8}$ are the only terms which are needed to absorb the ultraviolet (UV) divergences when the constituent quark fields $Q(x)$ are integrated out. ${ }^{3}$ If one wants to consider the case where photons are also integrated out then, to leading order in the chiral expansion and in the electric charge coupling $e$, the last term in the second line is also required to absorb further UV-divergences. Loops involving pion fields are subleading in the $1 / \mathrm{N}_{\mathrm{c}}$-expansion and hence, following the observation of Weinberg in ref. [15], the Lagrangian in eq. (3.1), when considered within the framework of the large- $\mathrm{N}_{\mathrm{c}}$ limit, is a renormalizable Lagrangian.

The $\pi \pi$ total cross sections in the $\mathrm{C} \chi \mathrm{QM}$ are then simply given by the corresponding $\pi \pi \rightarrow Q \bar{Q}$ cross sections. ${ }^{4}$ When restricted to chiral $\mathrm{SU}(2)$ and in the chiral limit where $m_{u}=m_{d}=0$, but still keeping the value of $g_{A}$ free, the terms of the interaction Lagrangian which are needed for the evaluation of these cross sections are $[Q=(U, D)]$ :

$$
\begin{aligned}
\mathcal{L}_{\text {int }}(x) \doteq & -i \frac{g_{A}}{\sqrt{2} f_{\pi}}\left[\bar{U} \gamma^{\mu} \gamma_{5} D \partial_{\mu} \pi^{+}+\bar{D} \gamma^{\mu} \gamma_{5} U \partial_{\mu} \pi^{-}+\frac{1}{\sqrt{2}}\left(\bar{U} \gamma^{\mu} \gamma_{5} U-\bar{D} \gamma^{\mu} \gamma_{5} D\right) \partial_{\mu} \pi^{0}\right] \\
& +i \frac{1}{4 f_{\pi}^{2}}\left\{\bar{U} \gamma^{\mu} U\left(\pi^{+} \partial_{\mu} \pi^{-}-\pi^{-} \partial_{\mu} \pi^{+}\right)+\bar{D} \gamma^{\mu} D\left(\pi^{-} \partial_{\mu} \pi^{+}-\pi^{+} \partial_{\mu} \pi^{-}\right)\right\} \\
& +i \frac{\sqrt{2}}{4 f_{\pi}^{2}}\left\{\bar{U} \gamma^{\mu} D\left(\pi^{0} \partial_{\mu} \pi^{+}-\pi^{+} \partial_{\mu} \pi^{0}\right)-\bar{D} \gamma^{\mu} U\left(\pi^{0} \partial_{\mu} \pi^{-}-\pi^{-} \partial_{\mu} \pi^{0}\right)\right\} \\
& -M_{Q} \sum_{Q=U, D} \bar{Q} Q .
\end{aligned}
$$

\footnotetext{
${ }^{3}$ We disregard couplings involving external fields alone to lowest order in the chiral expansion.

${ }^{4}$ For the sake of simplicity we omit in this first analysis the contribution of gluon interactions in the $\mathrm{C} \chi \mathrm{QM}$.
} 


\subsection{Calculation of the pion-pion total cross sections}

We fix the kinematics as follows:

$$
\pi(k)+\pi\left(k^{\prime}\right) \rightarrow Q(p)+\bar{Q}\left(p^{\prime}\right) .
$$

In the center of mass system:

$$
\begin{array}{ll}
k:\left(\frac{\sqrt{s}}{2},+\frac{\sqrt{s}}{2}, 0,0\right), & p:\left(\frac{\sqrt{s}}{2},+\frac{\sqrt{s-4 M_{Q}^{2}}}{2} \cos \theta,+\frac{\sqrt{s-4 M_{Q}^{2}}}{2} \sin \theta, 0\right) ; \\
k^{\prime}:\left(\frac{\sqrt{s}}{2},-\frac{\sqrt{s}}{2}, 0,0\right), \quad p^{\prime}:\left(\frac{\sqrt{s}}{2},-\frac{\sqrt{s-4 M_{Q}^{2}}}{2} \cos \theta,-\frac{\sqrt{s-4 M_{Q}^{2}}}{2} \sin \theta, 0\right) .
\end{array}
$$

The total cross sections for massless pions are then given by

$$
\sigma_{\pi \pi}^{\mathrm{tot}}(s)=\frac{1}{32 \pi s} \sqrt{1-\frac{4 M_{Q}^{2}}{s}} \int_{-1}^{+1} \mathrm{~d}(\cos \theta)|T(\pi \pi \rightarrow Q \bar{Q})|^{2}(s, \cos \theta),
$$

with $|T(\pi \pi \rightarrow Q \bar{Q})|^{2}(s, \cos \theta)$ receiving contributions from the relevant terms shown in the interaction Lagrangian in eq. (3.7).

\subsubsection{The $\pi^{+} \pi^{-}$total cross section}

The various contributions to this cross section come from the following sources:

- Terms proportional to $\frac{g_{A}^{4}}{f_{\pi}^{4}} N_{c} n_{f}$.

They come from the squared amplitudes $\pi^{+} \pi^{-} \rightarrow U \bar{U}$ and $\pi^{+} \pi^{-} \rightarrow D \bar{D}$ generated by the terms in the first line in eq. (3.7). They give a contribution

$$
\frac{g_{A}^{4}}{f_{\pi}^{4}} N_{c} n_{f} \Rightarrow \frac{N_{c} n_{f}}{192 \pi} \frac{g_{A}^{4}}{f_{\pi}^{4}} \sqrt{1-\frac{4 M_{Q}^{2}}{s}}\left(s+2 M_{Q}^{2}\right) .
$$

- Terms proportional to $\frac{1}{f_{\pi}^{4}} N_{c} n_{f}$.

They come from the squared amplitudes $\pi^{+} \pi^{-} \rightarrow U \bar{U}$ and $\pi^{+} \pi^{-} \rightarrow D \bar{D}$ generated by the terms in the second line of eq. (3.7). They give a contribution

$$
\frac{1}{f_{\pi}^{4}} N_{c} n_{f} \Rightarrow \frac{N_{c} n_{f}}{192 \pi} \frac{1}{f_{\pi}^{4}} \sqrt{1-\frac{4 M_{Q}^{2}}{s}}\left(s+2 M_{Q}^{2}\right) .
$$

- Terms proportional to $\frac{g_{A}^{2}}{f_{\pi}^{4}} N_{c} n_{f}$

They come from the the interference of the amplitudes $\pi^{+} \pi^{-} \rightarrow U \bar{U}$ and $\pi^{+} \pi^{-} \rightarrow$ $D \bar{D}$ in the first and second lines of eq. (3.7). They give a contribution

$$
\frac{g_{A}^{2}}{f_{\pi}^{4}} N_{c} n_{f} \Rightarrow-\frac{N_{c} n_{f}}{192 \pi} \frac{g_{A}^{2}}{f_{\pi}^{4}} \sqrt{1-\frac{4 M_{Q}^{2}}{s}} 2\left(s-10 M_{Q}^{2}\right) .
$$


The overall contribution to the $\pi^{+} \pi^{-}$total cross section is then

$$
\sigma_{\pi^{+} \pi^{-}}^{\text {tot }}(s)=\frac{N_{c} n_{f}}{192 \pi} \frac{M_{Q}^{2}}{f_{\pi}^{4}} \sqrt{1-\frac{4 M_{Q}^{2}}{s}}\left[\left(1-g_{A}^{2}\right)^{2} \frac{s}{M_{Q}^{2}}+2\left(1+10 g_{A}^{2}+g_{A}^{4}\right)\right] .
$$

We therefore find that there is a leading contribution of $\mathcal{O}(s)$ to this cross section which, however, vanishes for $g_{A}=1$. In other words, the $\pi^{+} \pi^{-}$scattering total cross section in the $\mathrm{C} \chi \mathrm{QM}$ violates the Froissart bound unless $g_{A}=1$.

Equation (3.14), for $g_{A}=1$, reduces to

$$
\sigma_{\pi^{+} \pi^{-}}^{\mathrm{tot}}(s)=\frac{N_{c} n_{f}}{32 \pi} \frac{4 M_{Q}^{2}}{f_{\pi}^{4}} \sqrt{1-\frac{4 M_{Q}^{2}}{s}},
$$

and, therefore, for large-s:

$$
\sigma_{\pi^{+} \pi^{-}}^{\mathrm{tot}}(s) \underset{s \rightarrow \infty}{\sim} \frac{N_{c} n_{f}}{32 \pi} \frac{4 M_{Q}^{2}}{f_{\pi}^{4}} .
$$

We then conclude that the leading contribution to the $\pi^{+} \pi^{-}$scattering total cross section for large $s$, in the $\mathrm{C} \chi \mathrm{QM}$ and provided $g_{A}=1$ with neglect of gluon corrections, goes to a constant. There is no factor $1 / m_{\pi}^{2}$ in this bound and it satisfies the Large$\mathrm{N}_{\mathrm{c}}$ counting rules; but it does not saturate the FM-bound.

\subsubsection{The $\pi^{ \pm} \pi^{0}$ total cross sections}

The various contributions to $\sigma_{\pi^{ \pm} \pi^{0}}^{\text {tot }}(s)$ come from the following sources:

- Terms proportional to $\frac{g_{A}^{4}}{f_{\pi}^{4}} N_{c} n_{f}$.

They come from the squared amplitudes generated by the terms in the first line in eq. (3.7). They give a contribution

$$
\frac{g_{A}^{4}}{f_{\pi}^{4}} N_{c} n_{f} \Rightarrow \frac{N_{c} n_{f}}{192 \pi} \frac{g_{A}^{4}}{f_{\pi}^{4}}\left\{\sqrt{1-\frac{4 M_{Q}^{2}}{s}}\left(s-11 M_{Q}^{2}\right)+48 \frac{M_{Q}^{4}}{s} \log \frac{1+\sqrt{1-\frac{4 M_{Q}^{2}}{s}}}{1-\sqrt{1-\frac{4 M_{Q}^{2}}{s}}}\right\} .
$$

- Terms proportional to $\frac{1}{f_{\pi}^{4}} N_{c} n_{f}$.

They come from the squared amplitudes generated by the terms in the second line of eq. (3.7). They give a contribution

$$
\frac{1}{f_{\pi}^{4}} N_{c} n_{f} \Rightarrow \frac{N_{c} n_{f}}{192 \pi} \frac{1}{f_{\pi}^{4}} \sqrt{1-\frac{4 M_{Q}^{2}}{s}}\left(s-2 M_{Q}^{2}\right) .
$$

- Terms proportional to $\frac{g_{A}^{2}}{f_{\pi}^{4}} N_{c} n_{f}$

They come from the the interference of amplitudes in the first and second lines of eq. (3.7). They give a contribution

$$
\frac{g_{A}^{2}}{f_{\pi}^{4}} N_{c} n_{f} \Rightarrow-\frac{N_{c} n_{f}}{192 \pi} \frac{g_{A}^{2}}{f_{\pi}^{4}} \sqrt{1-\frac{4 M_{Q}^{2}}{s}}\left(2 s-11 M_{Q}^{2}\right) .
$$


The overall contribution is then

$$
\begin{array}{r}
\sigma_{\pi^{ \pm} \pi^{0}}^{\mathrm{tot}}(s)=\frac{N_{c} n_{f}}{192 \pi} \frac{M_{Q}^{2}}{f_{\pi}^{4}}\left\{\sqrt{1-\frac{4 M_{Q}^{2}}{s}}\left[\frac{s}{M_{Q}^{2}}\left(1-g_{A}^{2}\right)\left[\left(1-g_{A}^{2}\right)-2\left(1+11 g_{A}^{2}\right)\right]\right]\right. \\
\left.+12 g_{A}^{4} \frac{4 M_{Q}^{2}}{s} \log \frac{1+\sqrt{1-\frac{4 M_{Q}^{2}}{s}}}{1-\sqrt{1-\frac{4 M_{Q}^{2}}{s}}}\right\} .
\end{array}
$$

We also find that there is a leading contributions of $\mathcal{O}(s)$ to the total cross section which, like in the $\pi^{+} \pi^{-}$case, vanishes for $g_{A}=1$. Therefore, the $\pi^{ \pm} \pi^{0}$ scattering total cross section in the $\mathrm{C} \chi \mathrm{QM}$ also violates the Froissart bound unless $g_{A}=1$.

Equation (3.20), for $g_{A}=1$, reduces to

$$
\sigma_{\pi^{ \pm} \pi^{0}}^{\mathrm{tot}}(s)=\frac{N_{c} n_{f}}{16 \pi} \frac{4 M_{Q}^{2}}{f_{\pi}^{4}} \frac{M_{Q}^{2}}{s} \log \frac{1+\sqrt{1-\frac{4 M_{Q}^{2}}{s}}}{1-\sqrt{1-\frac{4 M_{Q}^{2}}{s}}},
$$

which asymptotically, for large-s, goes as:

$$
\sigma_{\pi^{ \pm} \pi^{0}}^{\mathrm{tot}}(s) \underset{s \rightarrow \infty}{\sim} \frac{N_{c} n_{f}}{16 \pi} \frac{4 M_{Q}^{2}}{f_{\pi}^{4}} \frac{M_{Q}^{2}}{s} \log \frac{s}{M_{Q}^{2}},
$$

and is subleading when compared to the corresponding asymptotic behaviour of $\pi^{+} \pi^{-}$in eq. (3.16).

\subsubsection{The $\pi^{0} \pi^{0}$ total cross section}

The only term in the interaction Lagrangian in eq. (3.7) which contributes to this process is the one in the last term of the first line. Therefore, the contribution to $\sigma_{\pi^{0} \pi^{0}}^{\text {tot }}(s)$ only comes from:

- Terms proportional to $\frac{g_{A}^{4}}{f_{\pi}^{4}} N_{c} n_{f}$.

The overall contribution is then

$$
\sigma_{\pi^{0} \pi^{0}}^{\mathrm{tot}}(s)=\frac{N_{c} n_{f}}{32 \pi} \frac{4 M_{Q}^{2}}{f_{\pi}^{4}} g_{A}^{4}\left\{\sqrt{1-\frac{4 M_{Q}^{2}}{s}}-2 \frac{M_{Q}^{2}}{s} \log \frac{1+\sqrt{1-\frac{4 M_{Q}^{2}}{s}}}{1-\sqrt{1-\frac{4 M_{Q}^{2}}{s}}}\right\} .
$$

and contrary to the previous $\sigma_{\pi^{+} \pi^{-}}^{\text {tot }}(s)$ and $\sigma_{\pi^{ \pm} \pi^{0}}^{\text {tot }}(s)$ cases one does not need to fix $g_{A}=1$ to respect the Froissart bound.

Asymptotically, for large- $s$ and with $g_{A}=1$, it goes as:

$$
\sigma_{\pi^{0} \pi^{0}}^{\mathrm{tot}}(s) \underset{s \rightarrow \infty}{\sim} \frac{N_{c} n_{f}}{32 \pi} \frac{4 M_{Q}^{2}}{f_{\pi}^{4}}\left[1-2 \frac{M_{Q}^{2}}{s} \log \frac{s}{M_{Q}^{2}}\right],
$$

i.e. as a constant. 


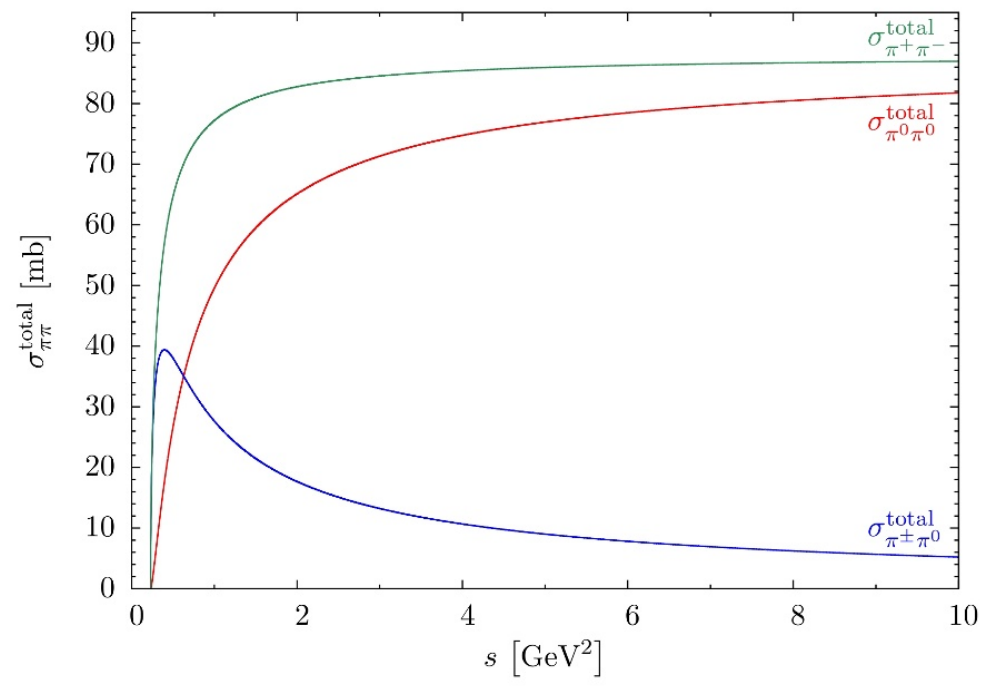

Figure 1. Total $\pi \pi$ cross sections in millibarns versus $s$ in $\mathrm{GeV}^{2}$.

The total $\pi \pi$ cross sections calculated above, with $g_{A}=1$, are plotted in figure 1 below. The higher curve corresponds to $\sigma_{\pi^{+} \pi^{-}}^{\text {total }}(s)$, the middle curve to $\sigma_{\pi^{0} \pi^{0}}^{\text {total }}(s)$ and the low curve to $\sigma_{\pi^{ \pm} \pi^{0}}^{\text {total }}(s)$. The cross sections are given in millibarn units and they are plotted versus $s$, the total center of mass squared energy, in $\mathrm{GeV}^{2}$. The constituent quark mass which we have used is the center value $M_{Q}=240 \mathrm{MeV}$ of the result $M_{Q}=(240 \pm 10) \mathrm{MeV}$ obtained in ref. [5]. Asymptotically, $\sigma_{\pi^{+} \pi^{-}}^{\text {tot }}(s)$ and $\sigma_{\pi^{0} \pi^{0}}^{\text {tot }}(s)$ have the same constant behaviour:

$$
\sigma_{\pi \pi}^{\mathrm{tot}}(s) \underset{s \rightarrow \infty}{\sim} \frac{N_{c} n_{f}}{32 \pi} \frac{4 M_{Q}^{2}}{f_{\pi}^{4}}, \quad \text { for } \pi^{+} \pi^{-} \text {and } \pi^{0} \pi^{0}
$$

while $\sigma_{\pi^{ \pm} \pi^{0}}^{\text {tot }}(s)$ falls as the difference between $\sigma_{\pi^{+} \pi^{-}}^{\text {tot }}(s)$ and $\sigma_{\pi^{0} \pi^{0}}^{\text {tot }}(s)$ (see eq. (3.22)) and it is subleading. We therefore find that the $\sigma_{\pi \pi}^{\text {tot }}(s)$ cross sections in the $\mathrm{C} \chi \mathrm{QM}$ satisfy the Froissart-Martin bound, but they do not saturate it. Very likely this is a drawback of the model, which ceases to be reliable at center of mass energy values of the order $\sqrt{s} \sim 2 \mathrm{GeV}$. It seems plausible that the rescattering of the constituent quarks in the presence of a gluonic background may be at the origin of an asymptotic black disc like behaviour, similar to the one observed in $p p$ and $p \bar{p}$ scattering [21-23], which provides the Froissart-Martin $\log ^{2} s$ enhancement.

For a discussion of the present experimental situation, phenomenological models and future prospects concerning $\sigma_{\pi \pi}^{\text {tot }}(s)$ cross sections at high energies see e.g. refs. $[24,25]$.

\subsection{Sum rules in the $\mathrm{C} \chi \mathrm{QM}$}

In the $\mathrm{C} \chi \mathrm{QM}$ the total $\pi^{+} \pi^{+}$cross section vanishes and therefore $\operatorname{Im} T^{2}(s, 0)=0$, which implies:

$$
\operatorname{Im} F_{1}(s, 0)=-\operatorname{Im} F_{2}(s, 0),
$$


and also the relations (with $g_{A}=1$ ):

$$
\begin{aligned}
\operatorname{Im} F_{2}(s, 0) & =\frac{s}{2}\left[\sigma_{\pi^{ \pm} \pi^{0}}^{\mathrm{tot}}+\sigma_{\pi^{0} \pi^{0}}^{\mathrm{tot}}\right](s)=\frac{s}{2} \sigma_{\pi^{+} \pi^{-}}^{\mathrm{tot}}(s) \\
& =\frac{s}{2} \frac{N_{c} n_{f}}{32 \pi} \frac{4 M_{Q}^{2}}{f_{\pi}^{4}} \sqrt{1-\frac{4 M_{Q}^{2}}{s}},
\end{aligned}
$$

and

$$
\begin{aligned}
\operatorname{Im} F_{3}(s, 0) & =\frac{s}{2}\left[3 \sigma_{\pi^{ \pm} \pi^{0}}^{\mathrm{tot}}-\sigma_{\pi^{0} \pi^{0}}^{\mathrm{tot}}\right](s) \\
& =\frac{s}{2} \frac{N_{c} n_{f}}{32 \pi} \frac{4 M_{Q}^{2}}{f_{\pi}^{4}}\left\{-\sqrt{1-\frac{4 M_{Q}^{2}}{s}}+8 \frac{M_{Q}^{2}}{s} \log \frac{1+\sqrt{1-\frac{4 M_{Q}^{2}}{s}}}{1-\sqrt{1-\frac{4 M_{Q}^{2}}{s}}}\right\} .
\end{aligned}
$$

Inserting these expressions in the r.h.s. of the dispersion relations in eq. (2.5) results in the sum rules:

$$
\frac{2}{\pi} \int_{4 M_{Q}^{2}}^{\infty} \frac{d s^{\prime}}{s^{\prime 3}} \operatorname{Im} F_{2,3}\left(s^{\prime}, 0\right)=\frac{1}{f_{\pi}^{4}} \frac{1}{3} \frac{N_{c} n_{f}}{16 \pi^{2}},
$$

which for $n_{f}=2$ reproduce the $\mathrm{C} \chi \mathrm{QM}$ results for $\operatorname{Re} F_{2,3}(s, 0)$ in eqs. $(2.7)$ and $(2.8)$, with $g_{A}=1$, and where [14]:

$$
2 l_{1}+3 l_{2}=-2 l_{1}+l_{2}=\frac{2}{3} \frac{N_{c}}{16 \pi^{2}} .
$$

In fact, the chiral expansions of the $\operatorname{Re} F_{1,2,3}(s, 0)$ amplitudes in the $\mathrm{C} \chi \mathrm{QM}$, evaluated with the dispersion relations in eqs. (2.5) and (2.6) are:

$$
\begin{aligned}
& \operatorname{Re} F_{1}(s, 0) \underset{s \rightarrow 0}{=}-\frac{s}{f_{\pi}^{2}}\left(1+\frac{N_{c} n_{f}}{240 \pi^{2}} \frac{s^{2}}{f_{\pi}^{2} M_{Q}^{2}}+\mathcal{O}\left(s^{4} / M_{Q}^{8}\right)\right), \\
& \operatorname{Re} F_{2}(s, 0) \underset{s \rightarrow 0}{=} \frac{s^{2}}{f_{\pi}^{4}} \frac{N_{c} n_{f}}{48 \pi^{2}}\left(1+\frac{1}{70} \frac{s^{2}}{M_{Q}^{4}}+\mathcal{O}\left(s^{4} / M_{Q}^{8}\right)\right), \\
& \operatorname{Re} F_{3}(s, 0) \underset{s \rightarrow 0}{=} \frac{s^{2}}{f_{\pi}^{4}} \frac{N_{c} n_{f}}{48 \pi^{2}}\left(1+\frac{1}{35} \frac{s^{2}}{M_{Q}^{4}}+\mathcal{O}\left(s^{4} / M_{Q}^{8}\right)\right) .
\end{aligned}
$$

\section{Pion-pion cross sections in Large- $\mathrm{N}_{c} \mathrm{QCD}$}

Let us now consider the pion-pion amplitudes within the framework of Large- $\mathrm{N}_{c}$ QCD. In this limit the $\operatorname{Im} T^{I}(s, 0)$ isospin amplitudes are composed of an infinite set of narrow states:

$$
\frac{1}{\pi} \operatorname{Im} T^{I}(s, 0)=\sum_{n=0}^{\infty}\left|F_{I, n}\right|^{2} \delta\left(s-M_{I, n}^{2}\right), \quad I=0,1 .
$$

The spacing of the narrow states, explored in Lattice Large- $\mathrm{N}_{\mathrm{c}}$ QCD simulations [26], is compatible with the Regge growth of the leading trajectories ${ }^{5}(n=0,1,2,3, \ldots)$ :

$$
M_{I=1, n}^{2}=\frac{1}{\alpha_{1}}\left(2 n+1-\alpha_{0}\right) \quad \text { and } \quad M_{I=0, n}^{2}=\frac{1}{\alpha_{1}}\left(2 n+2-\alpha_{0}\right),
$$

\footnotetext{
${ }^{5}$ See e.g. ref. [27].
} 
and, to a good approximation, with $\alpha_{1}$ and $\alpha_{0}$ as given by the Veneziano model [28]:

$$
\alpha_{1} \simeq \frac{1}{2 M_{\rho}^{2}} \quad \text { and } \quad \alpha_{0} \simeq 1 / 2 .
$$

With $M_{\rho}=770 \mathrm{MeV}$, this choice corresponds to:

$$
M_{\sigma}=1334 \mathrm{MeV} \sim f_{2}(1270) \text { and } \Lambda_{\rho}=\Lambda_{\sigma}=2 M_{\rho}
$$

In the absence of exotic trajectories and hence no poles with $I=2$, the optical theorem relates the imaginary parts of the forward isospin amplitudes to the total $\pi \pi$ cross sections as follows:

$$
\begin{aligned}
& \operatorname{Im} T^{1}(s, 0)=\frac{s}{2}\left[\sigma_{\pi^{+} \pi^{-}}^{\text {total }}(s)+3 \sigma_{\pi^{ \pm} \pi^{0}}^{\text {total }}(s)-\sigma_{\pi^{0} \pi^{0}}^{\text {total }}(s)\right]=\frac{s}{2} 4 \sigma_{\pi^{ \pm} \pi^{0}}^{\text {total }}(s) . \\
& \operatorname{Im} T^{0}(s, 0)=\frac{s}{2} 6 \sigma_{\pi^{0} \pi^{0}}^{\text {total }}(s) .
\end{aligned}
$$

Unfortunately, there is no information on the values of the residues $\left|F_{I, n}\right|$. As shown in ref. [5], demanding that the cross sections which define $\operatorname{Im} T^{I=1}(s, 0)$ and $\operatorname{Im} T^{I=0}(s, 0)$ in eqs. (4.5) and (4.6) grow asymptotically as the Froissart bound, requires the couplings $\left|F_{I, n}\right|^{2}$ to grow like $n \log ^{2} n$ as $n \rightarrow \infty$. This suggests as a possible set of Large- $\mathrm{N}_{\mathrm{c}}$ $\operatorname{Im} T^{I}(s, 0)$ amplitudes the simple Ansatz:

$$
\frac{1}{\pi} \operatorname{Im} T^{I}(s, 0)=\mathrm{C}_{\mathrm{I}} \sum_{n=0}^{\infty}\left(M_{I}^{2}+n \Lambda_{I}^{2}\right) \log ^{2}\left(\frac{M_{I}^{2}}{\Lambda_{I}^{2}}+n\right) \delta\left(s-M_{I}^{2}-n \Lambda_{I}^{2}\right),
$$

where:

$$
\Lambda_{I=1}=\Lambda_{\rho}, M_{I=1}=M_{\rho} \quad \text { and } \quad \Lambda_{I=0}=\Lambda_{\sigma}, M_{I=0}=M_{\sigma},
$$

and the $\mathrm{C}_{\mathrm{I}}$ are dimensionless constants. This is essentially the same Ansatz which was considered in ref. [5]. There is, however, an important extrapolation which one is making in assuming such an Ansatz, namely the fact that the $n \log ^{2} n$ pattern of the residues $\left|F_{I, n}\right|$ is valid not only at very high energies but also at low energies. Because of that extrapolation, the corresponding values that one obtains for the coefficients of the $\log ^{2} s$ behaviour of the total $\sigma_{\pi \pi}^{\text {total }}$ cross sections are likely to be a gross overestimation. In what follows, we propose a more elaborated Large- $\mathrm{N}_{\mathrm{c}}$ Ansatz where the residues $\left|F_{I, n}\right|$ start growing like $n$, as suggested by the Veneziano model, and only from a threshold $s_{0}$ onwards, where asymptotics sets in, are they modified by terms which grow as $n \log ^{2} n$. More precisely, we suggest the following Large- $\mathrm{N}_{\mathrm{c}}$ Ansatz:

$$
\frac{1}{\pi} \operatorname{Im} T^{I}(s, 0)=\sum_{n=0}^{\infty}\left(M_{I}^{2}+n \Lambda_{I}^{2}\right)\left[\mathrm{C}_{\mathrm{I}}^{\mathrm{V}}+\mathrm{C}_{\mathrm{I}}^{\mathrm{F}} \theta(n-N) \log ^{2}\left(\frac{M_{I}^{2}}{\Lambda_{I}^{2}}+n\right)\right] \delta\left(s-M_{I}^{2}-n \Lambda_{I}^{2}\right),
$$

with $N$ a sufficiently large integer so as to match the asymptotic threshold

$$
s_{0}=M_{I}^{2}+N \Lambda_{I}^{2},
$$


and the mass parameters $\Lambda_{I=1}=\Lambda_{\rho}, M_{I=1}=M_{\rho}$ and $\Lambda_{I=0}=\Lambda_{\sigma}, M_{I=0}=M_{\sigma}$ the same as in eq. (4.8). Equation (4.9) can also be written in the more convenient form:

$$
\begin{aligned}
\frac{1}{\pi} \operatorname{Im} T^{I}(s, 0)= & \mathrm{C}_{\mathrm{I}}^{\mathrm{V}} \sum_{n=0}^{\infty}\left(M_{I}^{2}+n \Lambda_{I}^{2}\right) \delta\left(s-M_{I}^{2}-n \Lambda_{I}^{2}\right) \\
& +\mathrm{C}_{\mathrm{I}}^{\mathrm{F}} \sum_{n=0}^{\infty}\left(s_{0}+n \Lambda_{I}^{2}\right) \log ^{2}\left(\frac{s_{0}}{\Lambda_{I}^{2}}+n\right) \delta\left(s-s_{0}-n \Lambda_{I}^{2}\right) .
\end{aligned}
$$

The Mellin transforms of infinite sums like the ones in eq. (4.11) have a close analytic form:

$$
\begin{aligned}
\Sigma^{I}(\xi) & =\int_{0}^{\infty} \mathrm{d}\left(\frac{s}{\Lambda_{I}^{2}}\right)\left(\frac{s}{\Lambda_{I}^{2}}\right)^{\xi-1} \frac{1}{\pi} \operatorname{Im} T^{I}(s, 0) \\
& =\mathrm{C}_{\mathrm{I}}^{\mathrm{V}} \zeta\left(-\xi, \frac{M_{I}^{2}}{\Lambda_{I}^{2}}\right)+\mathrm{C}_{\mathrm{I}}^{\mathrm{F}} \frac{d^{2}}{d \xi^{2}} \zeta\left(-\xi, \frac{s_{0}^{2}}{\Lambda_{I}^{2}}\right),
\end{aligned}
$$

where $\zeta\left(-\xi, \frac{M_{I}^{2}}{\Lambda_{I}^{2}}\right)$ is the Hurwitz function, a generalization of the Riemann zeta function, defined by the series:

$$
\zeta(\xi, v)=\sum_{n=0}^{\infty} \frac{1}{(n+v)^{\xi}}, \quad v \neq-1,-2,-3, \ldots
$$

and its analytic continuation. For $v=1$ it reduces to the Riemann zeta function. The asymptotic behaviour of $\frac{1}{\pi} \operatorname{Im} T^{I}(s, 0)$ for $s \rightarrow \infty$ is then governed by the inverse Mellin transform

$$
\frac{1}{\pi} \operatorname{Im} T^{I}(s, 0)=\frac{1}{2 \pi i} \int_{c_{\xi}-i \infty}^{c_{\xi}+i \infty} \mathrm{d} \xi\left(\frac{s}{\Lambda_{I}^{2}}\right)^{-\xi}\left[\mathrm{C}_{\mathrm{I}}^{\mathrm{V}} \zeta\left(-\xi, \frac{M_{I}^{2}}{\Lambda_{I}^{2}}\right)+\mathrm{C}_{\mathrm{I}}^{\mathrm{F}} \frac{d^{2}}{d \xi^{2}} \zeta\left(-\xi, \frac{s_{0}^{2}}{\Lambda_{I}^{2}}\right)\right] ;
$$

and more precisely, by the residues of the triple pole of the $\mathrm{C}_{\mathrm{I}}^{\mathrm{F}}$ term and the single pole of the $\mathrm{C}_{\mathrm{I}}^{\mathrm{V}}$ term of $\Sigma^{I}(\xi)$ at $\xi=-1$. This fixes the asymptotic behaviours of the total $\pi^{ \pm} \pi^{0}$ and $\pi^{0} \pi^{0}$ cross sections in the r.h.s. of eqs. (4.5) and (4.6) as follows:

$$
\begin{gathered}
\sigma_{\pi^{ \pm} \pi^{0}}^{\text {total }}(s) \underset{s \rightarrow \infty}{\sim} \frac{\pi}{2 \Lambda_{\rho}^{2}}\left(\mathrm{C}_{1}^{\mathrm{F}} \log ^{2} \frac{s}{s_{0}}+\mathrm{C}_{1}^{\mathrm{V}}\right), \\
\sigma_{\pi^{0} \pi^{0}}^{\text {total }}(s) \underset{s \rightarrow \infty}{\sim} \frac{\pi}{3 \Lambda_{\rho}^{2}}\left(\mathrm{C}_{0}^{\mathrm{F}} \log ^{2} \frac{s}{s_{0}}+\mathrm{C}_{0}^{\mathrm{V}}\right) .
\end{gathered}
$$

In order to learn something about the constants $\mathrm{C}_{\mathrm{I}}^{\mathrm{V}, \mathrm{F}}$ one can use information from the low energy behaviour of $\operatorname{Re} T^{I}(s, 0)$ using a Mellin-Barnes representation of the dispersion relations in eq. (2.5). This results in the expression [5]:

$$
\operatorname{Re} T^{I}(s, 0)=\frac{1}{2 \pi i} \int_{c_{\xi}-i \infty}^{c_{\xi}+i \infty} \mathrm{d} \xi\left(\frac{s}{\Lambda_{I}^{2}}\right)^{2-\xi} \Gamma(\xi) \Gamma(1-\xi)\left[1+\frac{\pi}{\Gamma\left(\frac{1}{2}+\xi\right) \Gamma\left(\frac{1}{2}-\xi\right)}\right] \Sigma^{I}(\xi-2),
$$

where $\Sigma^{I}(\xi-2)$ is the same Mellin transform as the one defined in eq. (4.12). The leading low energy behaviour of $\operatorname{Re} T^{I}(s, 0)$ is then governed by the leading singularity of the 
integrand in the r.h.s. of eq. (4.18) at the left of the fundamental strip $\left.c_{\xi}=\operatorname{Re} \xi \in\right] 0,1[$, i.e. at $\xi=0$, with the result

$$
\operatorname{Re} T^{I}(s, 0) \underset{s \rightarrow 0}{\sim} 2 \frac{s^{2}}{\Lambda_{I}^{4}}\left[\mathrm{C}_{\mathrm{I}}^{\mathrm{V}} \zeta\left(2, \frac{M_{I}^{2}}{\Lambda_{I}^{2}}\right)+\mathrm{C}_{\mathrm{I}}^{\mathrm{F}} \zeta^{\prime \prime}\left(2, \frac{s_{0}}{\Lambda_{I}^{2}}\right)\right] .
$$

This has to match the $\chi \mathrm{PT}$ expressions in eqs. (2.7) and (2.8) restricted to their Large- $\mathrm{N}_{\mathrm{c}}$ limit form i.e.,

$$
\begin{gathered}
\operatorname{Re} T^{1}(s, 0)=\operatorname{Re} F_{2}(s, 0)+\operatorname{Re} F_{3}(s, 0) \underset{s \rightarrow 0}{\sim} \frac{s^{2}}{f_{\pi}^{4}} 4 l_{2}\left(M_{\rho}\right), \\
\operatorname{Re} T^{0}(s, 0)=\frac{3}{2}\left[3 \operatorname{Re} F_{2}(s, 0)-\operatorname{Re} F_{3}(s, 0)\right] \underset{s \rightarrow 0}{\sim} \frac{s^{2}}{f_{\pi}^{4}} 12\left[l_{1}\left(M_{\rho}\right)+l_{2}\left(M_{\rho}\right)\right] .
\end{gathered}
$$

This matching gives a linear constraint between $\mathrm{C}_{\mathrm{I}}^{\mathrm{V}}, \mathrm{C}_{\mathrm{I}}^{\mathrm{F}}$ and the low-energy constants $l_{2}$ and $l_{1}+l_{2}$ :

$$
\begin{array}{r}
32 l_{2} \frac{M_{\rho}^{4}}{f_{\pi}^{4}}=\left[\mathrm{C}_{1}^{\mathrm{V}} \zeta\left(2, \frac{M_{\rho}^{2}}{\Lambda_{\rho}^{2}}\right)+\mathrm{C}_{1}^{\mathrm{F}} \zeta^{\prime \prime}\left(2, \frac{s_{0}}{\Lambda_{\rho}^{2}}\right)\right], \\
96\left(l_{1}+l_{2}\right) \frac{M_{\rho}^{4}}{f_{\pi}^{4}}=\left[\mathrm{C}_{0}^{\mathrm{V}} \zeta\left(2, \frac{M_{\sigma}^{2}}{\Lambda_{\sigma}^{2}}\right)+\mathrm{C}_{1}^{\mathrm{F}} \zeta^{\prime \prime}\left(2, \frac{s_{0}}{\Lambda_{\sigma}^{2}}\right)\right] .
\end{array}
$$

The behaviour of $\sigma_{\pi^{ \pm} \pi^{0}}^{\text {total }}(s)$ and $\sigma_{\pi^{0} \pi^{0}}^{\text {total }}(s)$ in eqs. (4.16) and (4.17) at the onset of the asymptotic threshold $s=s_{0}$ fixes the values of $\mathrm{C}_{1}^{\mathrm{V}}$ and $\mathrm{C}_{0}^{\mathrm{V}}$. We propose to identify this onset with the one of the asymptotic behaviours of $\sigma_{\pi^{ \pm} \pi^{0}}^{\text {total }}(s)$ and $\sigma_{\pi^{0} \pi^{0}}^{\text {total }}(s)$ in the $\mathrm{C} \chi \mathrm{QM}$, evaluated in eqs. (3.22) and (3.24). This results in the values:

$$
\begin{aligned}
\mathrm{C}_{1}^{\mathrm{V}} & =2 \Lambda_{\rho}^{2} \frac{N_{c} n_{f}}{16 \pi^{2} f_{\pi}^{2}} \frac{4 M_{Q}^{2}}{f_{\pi}^{2}} \frac{M_{Q}^{2}}{s_{0}} \log \frac{s_{0}}{M_{Q}^{2}}, \\
\mathrm{C}_{0}^{\mathrm{V}} & =2 \Lambda_{\rho}^{2} \frac{N_{c} n_{f}}{16 \pi^{2} f_{\pi}^{2}} \frac{16 M_{Q}^{2}}{f_{\pi}^{2}} .
\end{aligned}
$$

Given input values for $s_{0}$ and $l_{2}, l_{1}+l_{2}$, the four coefficients $\mathrm{C}_{1}^{\mathrm{V}}, \mathrm{C}_{1}^{\mathrm{F}} \mathrm{C}_{0}^{\mathrm{V}}, \mathrm{C}_{0}^{\mathrm{F}}$ are then fixed. Obviously the onset $s_{0}$ has to be larger than $M_{\rho}^{2}$ in the $I=1$ channel and larger than $M_{\sigma}^{2}$ in the $I=0$. Provided one can find solutions to these constraints, the result is then a phenomenological proposal which matches the low-energy behaviour of the the total cross sections $\sigma_{\pi^{ \pm} \pi^{0}}^{\text {total }}(s)$ and $\sigma_{\pi^{0} \pi^{0}}^{\text {total }}(s)$, as predicted by the $\mathrm{C} \chi \mathrm{QM}$, with their high-energy behaviour predicted by the Large- $\mathrm{N}_{c}$ Ansatz which we have described above. Numerical solutions to this proposal are discussed in the next section.

\section{$5 \quad$ Numerical results and conclusions}

Several remarks concerning the issues discussed in the previous sections are in order.

- First, with regards to the $\chi P T$ low energy constants $l_{1}$ and $l_{2}$.

A recent phenomenological determination of the $l_{1}$ and $l_{2}$ constants, renormalized at the $\rho$-mass scale, gives [29]:

$$
l_{1}^{\mathrm{r}}\left(M_{\rho}\right)=(-5.2 \pm 0.5) \times 10^{-3} \quad \text { and } \quad l_{2}^{\mathrm{r}}\left(M_{\rho}\right)=(4.0 \pm 1.5) \times 10^{-3} .
$$


For comparison, the values predicted by the low resonance saturation of the $l_{1}$ and $l_{2}$ constants are [30, 31]:

$$
l_{2}=\frac{1}{2} \frac{f_{\pi}^{2}}{M_{\rho}^{2}} \simeq 6.6 \times 10^{-3}, \quad \text { and } \quad l_{1}+l_{2}=\frac{1}{4} \frac{\left|c_{d}\right|^{2}}{M_{f_{0}}^{2}} \simeq 0.3 \times 10^{-3} .
$$

These are the values where $l_{2}$ is saturated by the $\rho$, with $f_{\pi} \simeq 0.088 \mathrm{GeV}$ i.e. the chiral limit value [32], and $l_{1}+l_{2}$ is saturated by the $f_{0}(983)$, with $\left|c_{d}\right| \simeq 3.2 \times 10^{-2} \mathrm{GeV}$ and $M_{f_{0}}=0.983 \mathrm{GeV}$ [30]. Within errors, these values compare rather well with the phenomenological determinations above. It is, however, far from clear that the $f_{0}(983)$ is a particle one should identify with a Large- $\mathrm{N}_{\mathrm{c}} \bar{q} q$ state [33]. We also recall that in the $\mathrm{C} \chi \mathrm{QM}$ (see eqs. (3.32) above) one finds:

$$
l_{2}=\frac{1}{3} \frac{N_{c}}{16 \pi^{2}}=6.3 \times 10^{-3} \text { and } l_{1}+l_{2}=\frac{1}{6} \frac{N_{c}}{16 \pi^{2}}=3.2 \times 10^{-3} .
$$

The $l_{2}$ values of the $\mathrm{C} \chi \mathrm{QM}$ and the $\rho$ saturation approximation compare very well. Unfortunately, the combination of low energy constants $l_{1}+l_{2}$ determined from experiments has a large error: $l_{1}+l_{2}=(-1.2 \pm 1.6) \times 10^{-3}$, which makes it difficult to extract useful information on the coefficients $\mathrm{C}_{0}^{\mathrm{V}}$ and $\mathrm{C}_{0}^{\mathrm{F}}$ using this phenomenological input.

- The size of $\zeta\left(2, \frac{M_{I}^{2}}{\Lambda_{I}^{2}}\right)$ and $\zeta^{\prime \prime}\left(2, \frac{s_{0}^{2}}{\Lambda_{I}^{2}}\right)$ in the r.h.s. of eq. (4.19).

Figure 2 below shows the shape of the factor $\zeta\left(2, \frac{M_{I}^{2}}{\Lambda_{I}^{2}}\right)$ for the range $0.2 \leq \frac{M_{I}^{2}}{\Lambda_{I}^{2}} \leq 1$. The particular values we are interested in are $\left(\Lambda_{\rho}=\Lambda_{\sigma}=2 M_{\rho}\right)$ :

$$
\zeta\left(2, \frac{M_{\rho}^{2}}{\Lambda_{\rho}^{2}}=\frac{1}{4}\right)=17.2 \quad \text { and } \quad \zeta\left(2, \frac{M_{\sigma}^{2}}{\Lambda_{\rho}^{2}}=0.75\right)=2.54 .
$$

figure 3 shows the shape of the factor $\zeta^{\prime \prime}\left(2, \frac{s_{0}}{\Lambda_{\rho}^{2}}\right)$ for the range $1 \leq \frac{s_{0}}{M_{\rho}^{2}} \leq 25$. The particular values we shall be using below are:

$$
\zeta^{\prime \prime}\left(2, \frac{\left(2.537 M_{\rho}\right)^{2}}{4 M_{\rho}^{2}}=1.61\right)=2.01 \quad \text { and } \quad \zeta^{\prime \prime}\left(2, \frac{\left(3.27 M_{\sigma}\right)^{2}}{4 M_{\rho}^{2}}=8.02\right)=1.34
$$

- Fixing the constants $\mathrm{C}_{1}^{\mathrm{V}}$ and $\mathrm{C}_{1}^{\mathrm{F}}$ and $\sigma_{\pi^{ \pm} \pi^{0}}^{\text {total }}$.

As a numerical example concerning the $I=1$ channel we show in figure 4 the total cross section $\sigma_{\pi^{ \pm} \pi^{0}}^{\text {total }}$ in millibarns versus $\sqrt{s}$ in $\mathrm{GeV}$ when $l_{2}$ is fixed to the center value of the phenomenological determination in eq. (5.1) and the choice of $s_{0}$ is tuned to $\sqrt{s_{0}}=2.537 M_{\rho}$ so as to obtain a slope of the $\log ^{2} \frac{s}{s_{0}}$ term in eq. (4.16) of the order of what is experimentally observed in the asymptotic behaviour of the total $p p$ total cross section [21-23]. The fact that this relatively simple model of Large- $\mathrm{N}_{\mathrm{c}}$ QCD we are considering can accommodate for such a solution with a reasonable value for $s_{0}$ which matches the asymptotic behaviour of the $\mathrm{C} \chi \mathrm{QM}$ is quite a remarkable fact. 


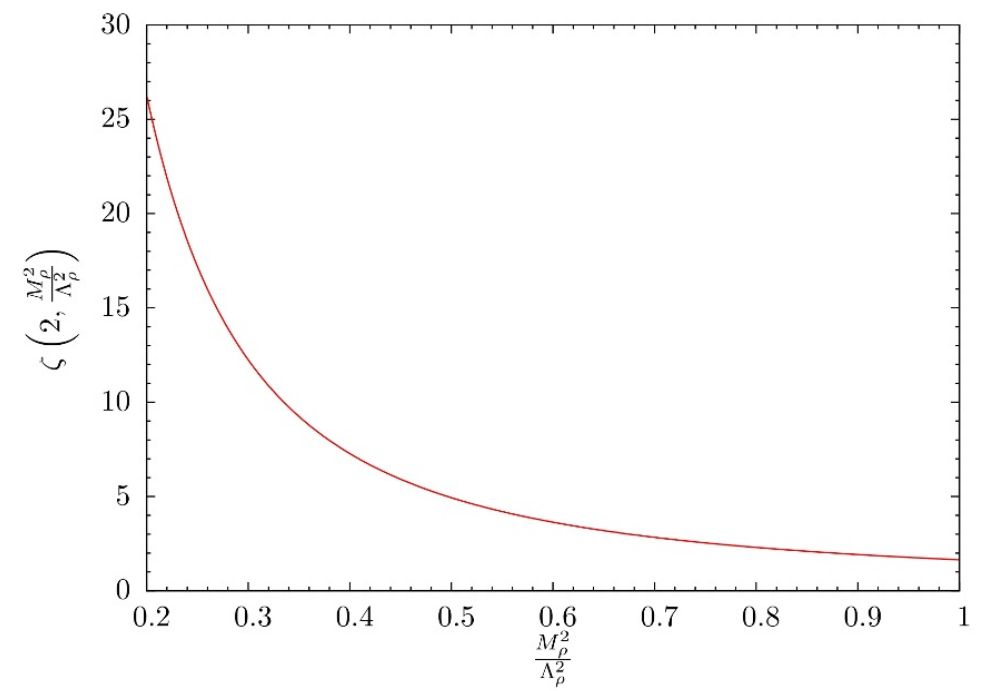

Figure 2. Shape of the factor $\zeta\left(2, \frac{M_{I}^{2}}{\Lambda_{I}^{2}}\right)$ in the r.h.s. of eqs. (4.19).

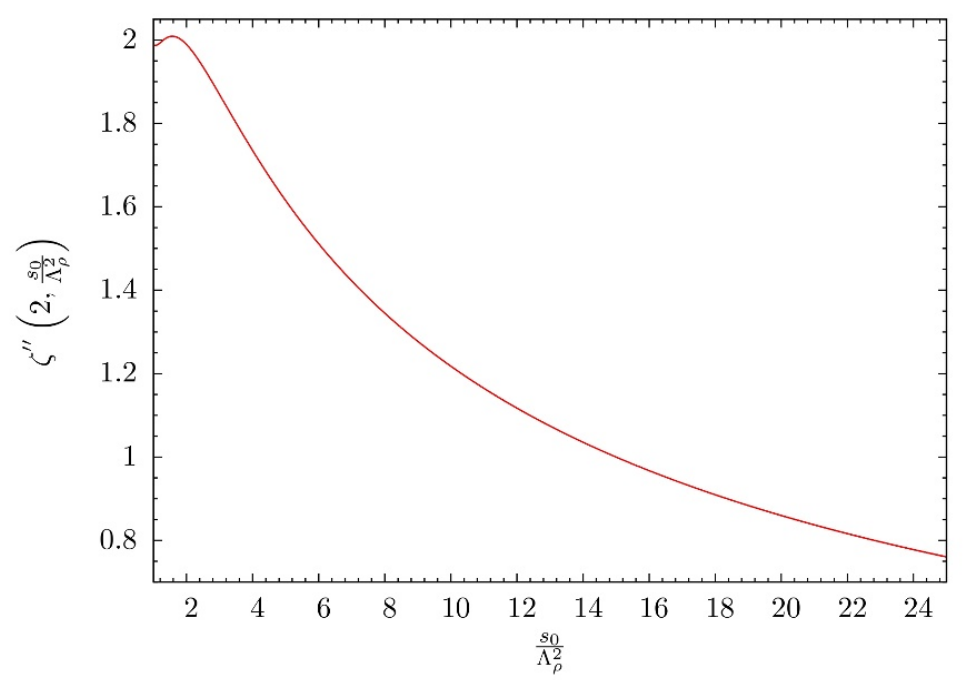

Figure 3. Shape of the factor $\zeta^{\prime \prime}\left(2, \frac{s_{0}}{\Lambda_{\rho}^{2}}\right)$ in the r.h.s. of eqs. (4.19).

- Fixing the constants $\mathrm{C}_{0}^{\mathrm{V}}$ and $\mathrm{C}_{0}^{\mathrm{F}}$ and $\sigma_{\pi^{ \pm} \pi^{0}}^{\text {total }}$.

As already mentioned, the phenomenological determination of $l_{1}+l_{2}$ has, unfortunately, a rather large error. On the other hand, the low resonance saturation value given in eq. (5.2), because of the questionable input of the scalar mass, is not reliable. The best option is to use the $\mathrm{C} \chi \mathrm{QM}$ determination in eq. (5.3), keeping in mind that this is a model-dependent input. Figure 5 shows the corresponding total cross section $\sigma_{\pi^{0} \pi^{0}}^{\text {total }}$ in millibarns versus $\sqrt{s}$ in $\mathrm{GeV}$ for a choice of $s_{0}$ tuned to $\sqrt{s_{0}}=3.27 M_{\sigma}$ so as to obtain a slope of the $\log ^{2} \frac{s}{s_{0}}$ term in eq. (4.17) of the order of what is experimentally observed in the asymptotic behaviour of the total $p p$ total cross section [21-23]. In spite of the fact that this is only an illustrative example of 


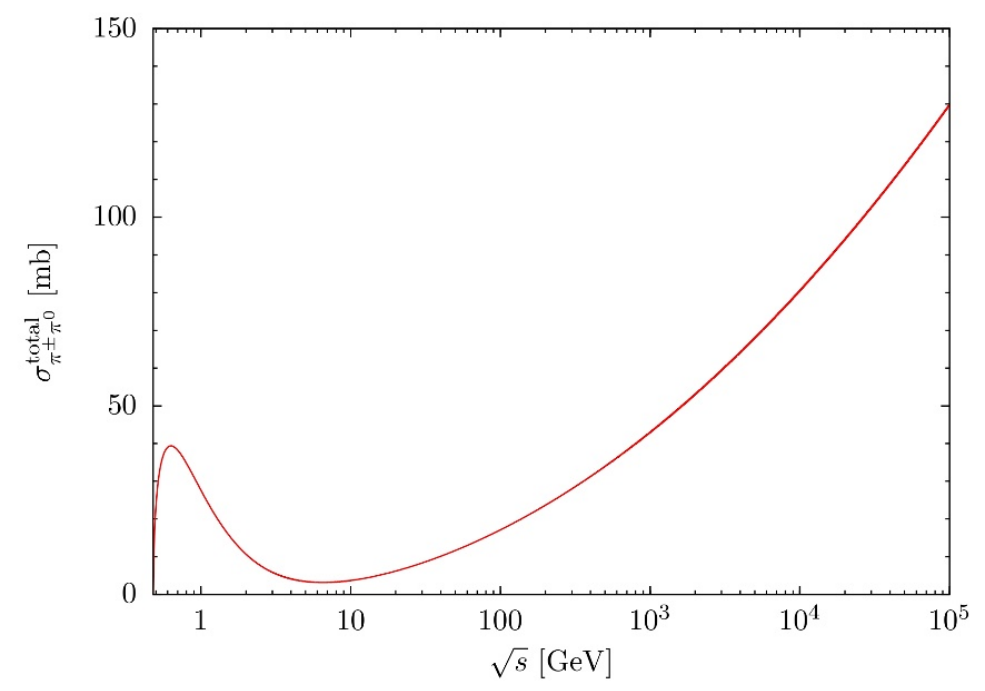

Figure 4. $\sigma_{\pi^{ \pm} \pi^{0}}^{\text {total }}$ in millibarns versus $\sqrt{s}$ in $\mathrm{GeV}$.

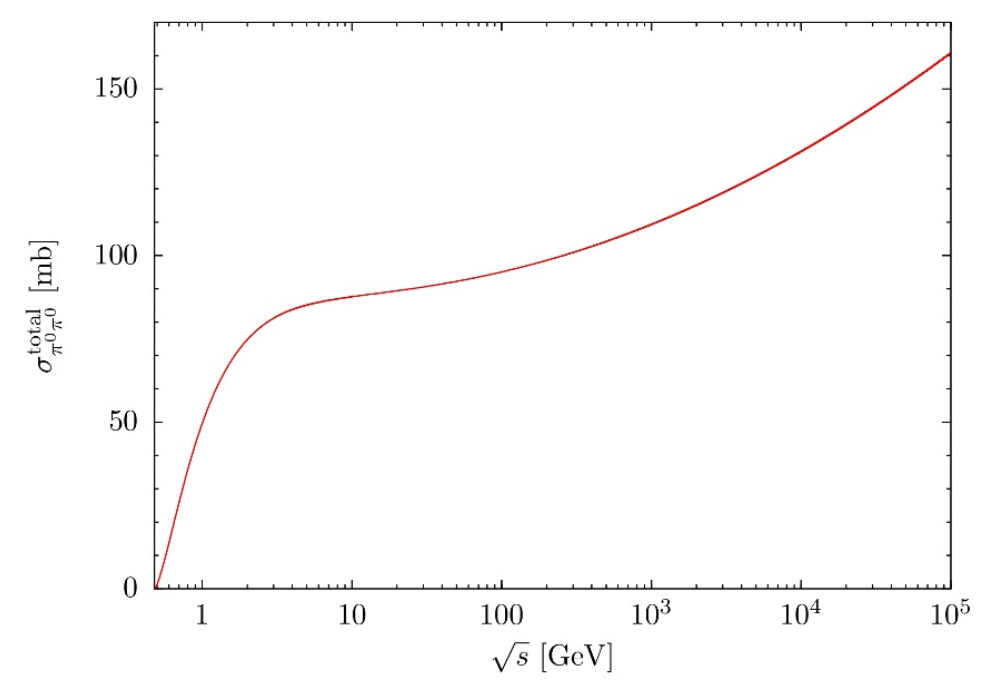

Figure 5. $\sigma_{\pi^{0} \pi^{0}}^{\text {total }}$ in millibarns versus $\sqrt{s}$ in $\mathrm{GeV}$.

a possible Large- $\mathrm{N}_{\mathrm{c}}$ Ansatz, it is quite remarkable that we can find a solution which respects the constraints discussed above with rather reasonable input values.

The main conclusion of this paper is the observation which we have discussed in section 2.1, namely the fact that -if the Froissart bound applies in QCD to $\pi \pi$ total cross sections- then the Lukaszuk-Martin coefficient $\pi / m_{\pi}^{2}$ of the $\log ^{2} s$ asymptotic behaviour cannot be the optimal one. The Lukaszuk-Martin coefficient violates both the QCD chiral behaviour and the QCD Large- $\mathrm{N}_{\mathrm{c}}$ counting rules.

Assuming that a Froissart-like $\log ^{2} s$ behaviour does apply to the total $\pi \pi$ scattering cross sections in QCD, we have then shown that it is possible to construct Large- $\mathrm{N}_{c}$ Ansatz which reproduces this behaviour with coefficients which are finite in the chiral limit and have the correct $\mathcal{O}\left(1 / \mathrm{N}_{\mathrm{c}}\right)$ counting in the Large- $\mathrm{N}_{\mathrm{c}}$ limit. We have constructed total cross 
sections for $\pi^{ \pm} \pi^{0}$ and $\pi^{0} \pi^{0}$ scattering which match the low-energy behaviour predicted by the $\mathrm{C} \chi \mathrm{QM}$ discussed in section 3 with the Large- $\mathrm{N}_{\mathrm{c}}$ Ansatz discussed in section 4 . They are shown in figures 4 and 5 above. The order of magnitude of these cross sections at very high energies are similar to the total $p p$ and $p \bar{p}$ scattering cross sections which are experimentally observed [21-23].

\section{Acknowledgments}

One of us (EdeR) is very grateful to José Ramón Pelaez for clarifying issues on the phenomenological determinations of the low energy constants $l_{1}$ and $l_{2}$ as well as for guidance on the Regge literature. Two of us (DG and EdeR) are also grateful to André Martin for correspondence and for challenging us in obtaining $\pi \pi$ cross sections in Large- $\mathrm{N}_{\mathrm{c}}$ QCD compatible with data on $p p$ and $p \bar{p}$ total cross sections at high energies.

EdeR thanks the support of the OCEVU Labex (ANR-11-LABX-0060) and the A*MIDEX project (ANR-11-IDEX-0001-02) funded by the "Investissements d'Avenir" French government program managed by the ANR. DG's work is supported in part by the EU under Contract MTRN-CT-2006-035482 (FLAVIAnet) and by MUIR, Italy, under Project 2005-023102. GV's research has been supported in part by the Spanish Government and ERDF funds from the EU Commission [grants FPA2007-60323, CSD2007-00042 (Consolider Project CPAN)]. GV also thanks Giancarlo D'Ambrosio and the INFN Sezione di Napoli for their hospitality and financial support.

Open Access. This article is distributed under the terms of the Creative Commons Attribution License (CC-BY 4.0), which permits any use, distribution and reproduction in any medium, provided the original author(s) and source are credited.

\section{References}

[1] M. Froissart, Asymptotic behavior and subtractions in the Mandelstam representation, Phys. Rev. 123 (1961) 1053 [INSPIRE].

[2] A. Martin, Extension of the axiomatic analyticity domain of scattering amplitudes by unitarity (I), Nuovo Cim. 42 A (1966) 930.

[3] L. Lukaszuk and A. Martin, Absolute upper bounds for $\pi \pi$ scattering, Nuovo Cim. 52 A (1967) 122.

[4] A. Martin and S. Roy, Froissart bound on total cross-section without unknown constants, Phys. Rev. D 89 (2014) 045015 [arXiv: 1306.5210] [inSPIRE].

[5] D. Greynat and E. de Rafael, The Froissart-Martin bound for $\pi \pi$ scattering in QCD, Phys. Rev. D 88 (2013) 034015 [arXiv: 1305.7045] [INSPIRE].

[6] A. Manohar and H. Georgi, Chiral quarks and the nonrelativistic quark model, Nucl. Phys. B 234 (1984) 189 [INSPIRE].

[7] B. Ananthanarayan, G. Colangelo, J. Gasser and H. Leutwyler, Roy equation analysis of $\pi \pi$ scattering, Phys. Rept. 353 (2001) 207 [hep-ph/0005297] [INSPIRE]. 
[8] S.M. Roy, Exact integral equation for pion pion scattering involving only physical region partial waves, Phys. Lett. B 36 (1971) 353 [InSPIRE].

[9] J. Gasser and H. Leutwyler, Chiral perturbation theory to one loop, Annals Phys. 158 (1984) 142 [INSPIRE].

[10] J. Gasser and H. Leutwyler, Chiral perturbation theory: expansions in the mass of the strange quark, Nucl. Phys. B 250 (1985) 465 [INSPIRE].

[11] A. De Rújula, H. Georgi and S. Glashow, Hadron masses in a gauge theory, Phys. Rev. D 12 (1975) 147 [INSPIRE].

[12] S. Weinberg, Phenomenological lagrangian, Physica 96 A (1984) 327.

[13] FLAG Working Group, S. Aoki et al., Review of lattice results concerning low energy particle physics, arXiv:1310.8555 [INSPIRE].

[14] D. Espriu, E. de Rafael and J. Taron, The QCD effective action at long distances, Nucl. Phys. B 345 (1990) 22 [Erratum ibid. B 355 (1991) 278-279] [InSPIRE].

[15] S. Weinberg, Pions in large-N quantum chromodynamics, Phys. Rev. Lett. 105 (2010) 261601 [arXiv: 1009.1537] [INSPIRE].

[16] D.B. Kaplan, Extended QCD, arXiv:1306.5818 [INSPIRE].

[17] Y. Nambu and G. Jona-Lasinio, Dynamical model of elementary particles based on an analogy with superconductivity. 1, Phys. Rev. 122 (1961) 345 [InSPIRE].

[18] J. Bijnens, C. Bruno and E. de Rafael, Nambu-Jona-Lasinio like models and the low-energy effective action of QCD, Nucl. Phys. B 390 (1993) 501 [hep-ph/9206236] [INSPIRE].

[19] E. de Rafael, The constituent chiral quark model revisited, Phys. Lett. B 703 (2011) 60 [arXiv: 1107.0226] [INSPIRE].

[20] D. Greynat and E. de Rafael, Hadronic contributions to the muon anomaly in the constituent chiral quark model, JHEP 07 (2012) 020 [arXiv:1204.3029] [INSPIRE].

[21] M.M. Block and F. Halzen, New experimental evidence that the proton develops asymptotically into a black disk, Phys. Rev. D 86 (2012) 051504 [arXiv:1208.4086] [INSPIRE].

[22] M.J. Menon and P.V.R. Silva, A study on analytic parametrizations for proton-proton cross-sections and asymptotia, J. Phys. G 40 (2013) 125001.

[23] M.M. Block and F. Halzen, New experimental evidence that the proton develops asymptotically into a black disk, Phys. Rev. D 86 (2012) 051504 [arXiv:1208.4086] [INSPIRE].

[24] J. Pelaez and F. Yndurain, Regge analysis of pion pion (and pion kaon) scattering for energy $S^{1 / 2}>1.4$ GeV, Phys. Rev. D 69 (2004) 114001 [hep-ph/0312187] [INSPIRE].

[25] A. Grau, G. Pancheri, O. Shekhovtsova and Y.N. Srivastava, Modeling pion and proton total cross-sections at LHC, Phys. Lett. B 693 (2010) 456 [arXiv: 1008.4119] [InSPIRE].

[26] G. Bali et al., The meson spectrum in large-N QCD, PoS (Confinement X) 278 [arXiv: 1302.1502] [INSPIRE].

[27] A.V. Anisovitch, V.V. Anisovitch and A.V. Sarantsev, Systematics of $q$ anti-q states in the $\left(n, M^{2}\right)$ and $\left(J, M^{2}\right)$ planes, Phys. Rev. D 62 (2000) 051502 [hep-ph/0003113] [INSPIRE]. 
[28] G. Veneziano, Construction of a crossing-symmetric, Regge behaved amplitude for linearly rising trajectories, Nuovo Cim. 57 A (1968) 190.

[29] J. Nebreda, J.R. Peláez and G. Rios, Determination of chiral perturbation theory low energy constants from a precise determination of $\pi \pi$ scattering threshold parameters, Phys. Rev. D 88 (2013) 054001 [arXiv: 1205.4129] [INSPIRE].

[30] G. Ecker, J. Gasser, A. Pich and E. de Rafael, The role of resonances in chiral perturbation theory, Nucl. Phys. B 321 (1989) 311 [InSPIRE].

[31] Y. Novozhilov, A. Pronko and D. Vassilevich, Quark path integral with diquark currents and its anomalies, Phys. Lett. B 321 (1994) 425 [INSPIRE].

[32] G. Ecker, Facets of chiral perturbation theory, Nucl. Phys. Proc. Suppl. 245 (2013) 1 [arXiv: 1310.2084] [INSPIRE].

[33] J.R. Pelaez and G. Rios, Nature of the $f_{0}(600)$ from its $N_{c}$ dependence at two loops in unitarized chiral perturbation theory, Phys. Rev. Lett. 97 (2006) 242002 [hep-ph/0610397] [INSPIRE]. 